\title{
Multiple Hurwitz Zeta Functions
}

\author{
M. Ram Murty and Kaneenika Sinha
}

ABSTRACT. After giving a brief overview of the theory of multiple zeta func-
tions, we derive the analytic continuation of the multiple Hurwitz zeta function
\[ \zeta\left(s_{1}, \ldots, s_{r} ; x_{1}, \ldots, x_{r}\right):=\sum_{n_{1}>n_{2}>\cdots>n_{r} \geq 1} \frac{1}{\left(n_{1}+x_{1}\right)^{s_{1}} \cdots\left(n_{r}+x_{r}\right)^{s_{r}}} \]

using the binomial theorem and Hartogs' theorem. We also consider the cognate multiple $L$-functions,

$$
L\left(s_{1}, \ldots, s_{r} ; \chi_{1}, \ldots, \chi_{r}\right)=\sum_{n_{1}>n_{2}>\cdots>n_{r} \geq 1} \frac{\chi_{1}\left(n_{1}\right) \chi_{2}\left(n_{2}\right) \cdots \chi_{r}\left(n_{r}\right)}{n_{1}^{s_{1}} n_{2}^{s_{2}} \cdots n_{r}^{s_{r}}}
$$

where $\chi_{1}, \ldots, \chi_{r}$ are Dirichlet characters of the same modulus.

\section{Introduction}

In a fundamental paper written in 1859, Riemann [34] introduced his celebrated zeta function that now bears his name and indicated how it can be used to study the distribution of prime numbers. This function is defined by the Dirichlet series

$$
\zeta(s)=\sum_{n=1}^{\infty} \frac{1}{n^{s}}
$$

in the half-plane $\operatorname{Re}(s)>1$. Riemann proved that $\zeta(s)$ extends analytically for all $s \in \mathbb{C}$, apart from $s=1$ where it has a simple pole with residue 1 . He also established the remarkable functional equation

$$
\pi^{-\frac{s}{2}} \zeta(s) \Gamma\left(\frac{s}{2}\right)=\pi^{-(1-s) / 2} \zeta(1-s) \Gamma\left(\frac{1-s}{2}\right)
$$

and made the famous conjecture (now called the Riemann hypothesis) that if $\zeta(s)=$ 0 and $0<\operatorname{Re}(s)<1$, then $\operatorname{Re}(s)=\frac{1}{2}$. This is still unproved.

In 1882, Hurwitz [20] defined the "shifted" zeta function, $\zeta(s ; x)$ by the series

$$
\sum_{n=0}^{\infty} \frac{1}{(n+x)^{s}}
$$

for any $x$ satisfying $0<x \leq 1$. Thus, $\zeta(s ; 1)=\zeta(s)$.

1991 Mathematics Subject Classification. Primary 11M41, Secondary 11M35.

Key words and phrases. Hurwitz zeta function, multiple zeta functions.

Research of the first author was partially supported by an NSERC grant. 
This Hurwitz zeta function, originally defined for $\operatorname{Re}(s)>1$, can also be extended analytically for all $s \in \mathbb{C}$, apart from $s=1$, where it has a simple pole with residue 1 . In his study of $\zeta(s ; x)$, Hurwitz was motivated by the problem of analytic continution of Dirichlet $L$-functions. For any Dirichlet character $\chi$ (mod $q)$, we may write

$$
L(s, \chi):=\sum_{n=1}^{\infty} \frac{\chi(n)}{n^{s}}=q^{-s} \sum_{a(\bmod q)} \chi(a) \zeta(s ; a / q)
$$

so that the analytic continuation for the Hurwitz zeta function gives us the same for the $L(s, \chi)$. Thus, Hurwitz confined his attention for $x$ rational lying in the interval $(0,1)$.

In this paper, we will indicate a method by which the analytic continuation of $\zeta(s ; x)$ can be easily derived from the continuation of $\zeta(s)$. In fact, our derivation also gives the analytic continuation of $\zeta(s)$. The continuation of $\zeta(s ; x)$ can be enlarged to complex values of $x$ in the cut complex plane $\mathbb{C} \backslash(-\infty, 0]$. For instance, we will show that for such complex $z$, the formula

$$
\zeta(1-k, z)=-\frac{B_{k}(z)}{k}
$$

is still valid for any natural number $k \geq 2$. Here $B_{k}(z)$ is the $k$-th Bernoulli polynomial. This formula is well-known for $z$ real with $0<z \leq 1$.

The method of analytic continuation we will outline is applicable to a wider context of the theory of multiple Hurwitz zeta functions and more generally to the problem of analytic continuation of series of the form

$$
\sum_{n_{1}>n_{2}>\cdots n_{r} \geq 1} \frac{a_{n_{1}} \cdots a_{n_{r}}}{\left(n_{1}+x_{1}\right)^{s_{1}} \cdots\left(n_{r}+x_{r}\right)^{s_{r}}} .
$$

Such series have also been studied by various authors (see [10] and [28], for example). As David Bradley pointed out to us, our method appears in a nascent form in a paper of Stark [36] where it is used to rederive Dirichlet's class number formula.

The study of special values of the Riemann zeta function has motivated the study of multiple zeta values (MZV's) or the multiple zeta functions defined as:

$$
\zeta\left(s_{1}, \ldots, s_{r}\right)=\sum_{n_{1}>n_{2}>\cdots>n_{r} \geq 1} \frac{1}{n_{1}^{s_{1}} \cdots n_{r}^{s_{r}}} .
$$

Originally, the special values when $s_{1} \geq 2$ and $s_{2}, \ldots, s_{r} \geq 1$ with $s_{i}$ integral for $1 \leq i \leq r$, have been the main focus of attention. In this situation, the sum $s_{1}+s_{2}+\cdots+s_{r}$ is called the weight of $\zeta\left(s_{1}, \ldots, s_{r}\right)$ and $r$ is called its length (or sometimes depth).

The reader may find several excellent expositions of the theory of MZV's in the literature. There are two aspects of the theory: algebraic and analytic. For the algebraic aspect, we recommend Cartier [11], Waldschmidt [39] or Zudilin [43]. For the analytic side, we suggest Matsumoto [25]. Even though some special cases of the theory were studied by Euler, their formal definition and study emerges in the work of Hoffman [18] as well as Zagier [41].

It is easy to see that this series converges absolutely for $\operatorname{Re}\left(s_{i}\right) \geq 1$ for $2 \leq i \leq r$ and $\operatorname{Re}\left(s_{1}\right)>1$ and so, one can consider the problem of analytic continuation.

This question has been studied by several authors. The earliest work seems to be that of Atkinson [6] in his studies of the mean values of the Riemann zeta 
function. He derived the analytic continuation of $\zeta\left(s_{1}, s_{2}\right)$ using the Poisson summation formula. Perhaps not aware of this work, Matsuoka [29] in 1982 derived the analytic continuation of $\zeta(s, 1)$ and Apostol and $\mathrm{Vu}[\mathbf{5}]$ in 1984 again studied the case $r=2$. In both papers, the main tool was the Euler-Maclaurin summation formula. Fixing $s_{2}, \ldots, s_{r}$, Arakawa and Kaneko [3] showed that $\zeta\left(s_{1}, s_{2}, \ldots, s_{r}\right)$ can be analytically continued as a function of $s_{1}$ to the whole complex $s_{1}$-plane. The general case of continuation to $\mathbb{C}^{r}$ seems to have been independently addressed by Zhao [42] and Akiyama, Egami and Tanigawa [1].

In this paper, we will study the multiple Hurwitz zeta function:

$$
\zeta\left(s_{1}, \ldots, s_{r} ; x_{1}, \ldots, x_{r}\right):=\sum_{n_{1}>n_{2}>\cdots>n_{r} \geq 1} \frac{1}{\left(n_{1}+x_{1}\right)^{s_{1}} \cdots\left(n_{r}+x_{r}\right)^{s_{r}}},
$$

as well as the cognate multiple $L$-functions of Goncharov [17]:

$$
L\left(s_{1}, \ldots, s_{r} ; \chi_{1}, \ldots, \chi_{r}\right):=\sum_{n_{1}>n_{2}>\cdots>n_{r} \geq 1} \frac{\chi_{1}\left(n_{1}\right) \chi_{2}\left(n_{2}\right) \cdots \chi_{r}\left(n_{r}\right)}{n_{1}^{s_{1}} n_{2}^{s_{r}} \cdots n_{r}^{s_{r}}},
$$

where $\chi_{1}, \chi_{2}, \ldots, \chi_{r}$ are Dirichlet characters (of necessarily the same modulus). The analytic theory of the multiple Hurwitz zeta function has been studied by Akiyama and Ishikawa [2], who derived the meromorphic continuation to $\mathbb{C}^{r}$. The authors in [2] used the Euler-Maclaurin summation formula to obtain their results. Our goal in this paper is to show that this in fact is a simple consequence of the meromorphic continuation of the multiple zeta function. We will not be able to discuss the interesting algebraic and combinatorial aspects of multiple Hurwitz zeta functions and consequently refer the reader to $[\mathbf{9}]$ and $[\mathbf{3 0}]$.

We should point out that terminology and notation vary in the literature concerning multiple zeta functions. For example, some authors have the summations in the reverse order with $n_{1}<n_{2}<\cdots<n_{r}$. With our notation, there are some advantages. For instance, one can show [25] that the multiple Hurwitz zeta function converges absolutely in the region defined by

$$
\operatorname{Re}\left(s_{1}\right)>1, \quad \operatorname{Re}\left(s_{1}+s_{2}\right)>2, \quad \cdots \quad \operatorname{Re}\left(s_{1}+\cdots+s_{r}\right)>r .
$$

In some papers, MZV's are also called Euler-Zagier sums.

\section{An overview of MZV's}

The study of MZVs has opened up fascinating connections to physics and other branches of mathematics. For an elaboration of these connections, the reader may consult Cartier [12] or Zagier [41] for the details. The theory has classical roots. For example, the 1734 theorem of Euler states that

$$
2 \zeta(2 k)=(-1)^{k-1}(2 \pi)^{2 k} \frac{B_{2 k}}{(2 k) !}
$$

where $B_{n}$ designates the $n$-th Bernoulli number. He deduced this from the fact that

$$
\frac{\sin \pi t}{\pi t}=\prod_{n=1}^{\infty}\left(1-\frac{t^{2}}{n^{2}}\right)
$$


By comparing coefficients of $t^{2 m}$ of both sides of this formula, we see immediately that

$$
\zeta(\underbrace{2, \ldots, 2}_{m})=\frac{\pi^{2 m}}{(2 m+1) !} .
$$

Another pretty formula, conjectured by Zagier [41], is

$$
\zeta(\underbrace{3,1, \cdots, 3,1}_{2 n})=\frac{2 \pi^{4 n}}{(4 n+2) !} .
$$

This was proved by Borwein, Bradley, Broadhurst and Lisonek [9]. Their proof was based on the identity

$$
1+\sum_{n=1}^{\infty} \zeta(\underbrace{3,1, \cdots, 3,1}_{2 n}) t^{4 n}=\frac{\cosh \pi t-\cos \pi t}{\pi^{2} t^{2}} .
$$

In this repertoire of formulas, we can also observe that the infinite product

$$
\cos \pi t=\prod_{n=1}^{\infty}\left(1-\frac{t^{2}}{(n-1 / 2)^{2}}\right)
$$

leads to the following special value of the multiple Hurwitz zeta function:

$$
\zeta(\underbrace{2, \ldots, 2}_{m} ; \underbrace{-1 / 2, \ldots,-1 / 2}_{m})=\frac{\pi^{2 m}}{(2 m) !} .
$$

Recently, many identities between MZV's have been found (see for example, $[43]$ or $[8]$ ). One expects that all of these identities can be "explained" by a theory we will briefly outline below.

For one thing, it is hoped that the study of the MZV's will enhance our understanding of $\zeta(2 k+1)$, for $k=1,2, \ldots$ In these cases, we know from the 1978 work of Apéry [4] that $\zeta(3)$ is irrational, and from the recent 2000 work of Rivoal [35], Ball [7] and Zudilin [44] that infinitely many of these are irrational. One even has some quantitative information. For example, it is known that the $\mathbb{Q}$-space spanned by $\zeta(3), \zeta(5), \ldots, \zeta(a)$ has dimension $\gg \log a$. Recently, Zudilin has shown that one of $\zeta(5), \zeta(7), \zeta(9), \zeta(11)$ is irrational and that for some $j \in[5,69]$, the three numbers $1, \zeta(3), \zeta(j)$ are linearly independent over $\mathbb{Q}$. It is conjectured that the numbers

$$
\pi, \zeta(3), \zeta(5), \ldots
$$

are algebraically independent over $\mathbb{Q}$.

On the other hand, there are integral formulas for the MZV's describing them as "periods." A formula of Chen allows us to express a product of such integrals again as a linear combination of "shuffles" of the integral. One can also derive the so-called "stuffle" relations arising from the series representations of MZV's. One conjectures that these essentially exhaust all possible relations among the MZV's. We give a more precise description below.

For example, for $k$ a natural number greater than 1 , we have the $k$-dimensional integral

$$
\zeta(k)=\int_{1>t_{1}>\cdots>t_{k}>0} \frac{d t_{1}}{t_{1}} \cdots \frac{d t_{k-1}}{t_{k-1}} \frac{d t_{k}}{1-t_{k}},
$$


as is easily verified by direct integration. Also,

$$
\zeta(2,1)=\int_{1>t_{1}>t_{2}>t_{3}>0} \frac{d t_{1}}{t_{1}} \cdot \frac{d t_{2}}{1-t_{2}} \cdot \frac{d t_{3}}{1-t_{3}} .
$$

Following Chen [13], we define inductively the iterated integral of differential forms $\phi_{1}, \ldots, \phi_{m}$ on $[0,1]$ as

$$
\int_{0}^{1} \phi_{1} \cdots \phi_{m}:=\int_{0}^{1} \phi_{1}(t) \int_{0}^{t} \phi_{2} \cdots \phi_{m} .
$$

With this convention, we define the two differential forms,

$$
\omega_{0}=\frac{d t}{t}, \quad \omega_{1}=\frac{d t}{1-t},
$$

then the above two formulas can be written as

$$
\zeta(k)=\int_{0}^{1} \omega_{0}^{k-1} \omega_{1},
$$

and

$$
\zeta(2,1)=\int_{0}^{1} \omega_{0} \omega_{1}^{2},
$$

respectively. More generally, we have the Drinfeld-Kontsevich integral:

$$
\zeta\left(s_{1}, \ldots, s_{r}\right)=\int_{0}^{1} \omega_{0}^{s_{1}-1} \omega_{1} \cdots \omega_{0}^{s_{k}-1} \omega_{1}
$$

The product of two such integrals is again a linear combination of such integrals given by the "shuffle product."

To be precise, we review the notion of the shuffle product. Let $X$ be a finite alphabet and let $X^{*}$ be the set of words it generates. The length of a word is the number of letters it has. The algebra generated by $X^{*}$ over $\mathbb{Q}$ will be denoted $\mathbb{Q}\langle X\rangle$ and this is just the polynomial algebra in the non-commuting variables of $X$.

We define the "shuffle product" of two words $x_{1} \cdots x_{m}$ and $x_{m+1} \cdots x_{m+n}$ as

$$
\left(x_{1} \cdots x_{m}\right) \amalg\left(x_{m+1} \cdots x_{m+n}\right)=\sum_{\sigma \in \Sigma_{m, n}} x_{\sigma(1)} \cdots x_{\sigma(m+n)}
$$

where $\Sigma_{m, n}$ is the set of all permutations $\sigma$ on $\{1,2, \cdots, m+n\}$ satisfying

$$
\sigma(1)<\sigma(2)<\cdots<\sigma(m) \text { and } \sigma(m+1)<\sigma(m+2)<\cdots<\sigma(m+n) .
$$

Thus, $\Sigma_{m, n}$ has

$$
\left(\begin{array}{c}
m+n \\
m
\end{array}\right)
$$

elements. The terminology is suggested by the usual riffle shuffling of a deck of $m+n$ cards cut into two parts of $m$ cards and $n$ cards. Thus the summation is over all the possible "shuffles." Here is the formal definition. If $e$ denotes the empty word, we define the shuffle product inductively:

$$
e \amalg w=w \amalg e=w
$$

for all $w \in X^{*}$ and for $x, y \in X, u, v \in X^{*}$, we set

$$
(x u) \amalg(y v)=x(u \amalg y v)+y(x u \amalg v) .
$$

This can be thought in the following way. When shuffling $x u$ and $y v$, either $x$ goes first and we shuffle $u$ and $y v$ or $y$ goes first and we shuffle $x u$ and $v$. This rule is extended by linearity to all of $\mathbb{Q}\langle X\rangle$. 
How this relates to MZV's can be explained as follows. Let $X=\{x, y\}$ and consider $\mathbb{Q}\langle X\rangle$. To each tuple $\alpha=\left(s_{1}, \ldots, s_{r}\right)$ we associate the word

$$
w_{\alpha}=x^{s_{1}-1} y x^{s_{2}-1} y \cdots x^{s_{r}-1} y
$$

and define $\zeta\left(w_{\alpha}\right)$ to be $\zeta\left(s_{1}, \ldots, s_{r}\right)$. We extend the definition of $\zeta$ by linearity to the subalgebra $H$ of $\mathbb{Q}\langle X\rangle$ generated by all the words of the form $w_{\alpha}$.

Then, one can show that for $w_{\alpha}$ and $w_{\beta}$ (not necessarily of the same length), that

$$
\zeta\left(w_{\alpha}\right) \zeta\left(w_{\beta}\right)=\zeta\left(w_{\alpha} \amalg w_{\beta}\right) .
$$

These are called the shuffle relations among the MZV's. There are other relations among the MZV's that are not included in the shuffle relations described above. These arise from the series representations. For example, it is not hard to see that

$$
\zeta\left(s_{1}\right) \zeta\left(s_{2}\right)=\zeta\left(s_{1}, s_{2}\right)+\zeta\left(s_{2}, s_{1}\right)+\zeta\left(s_{1}+s_{2}\right)
$$

since the product on the left is a double sum

$$
\sum_{n_{1}=1}^{\infty} \sum_{n_{2}=1}^{\infty} \frac{1}{n_{1}^{s_{1}}} \frac{1}{n_{2}^{s_{2}}}
$$

which can be decomposed according as $n_{1}>n_{2}, n_{2}>n_{1}$ and $n_{1}=n_{2}$. In a similar way, we see that the product

$$
\zeta\left(s_{1}\right) \zeta\left(s_{2}, \ldots, s_{r}\right)=\sum_{n_{1}, n_{2}<n_{3}<\cdots n_{r}} \frac{1}{n_{1}^{s_{1}} n_{2}^{s_{2}} \cdots n_{r}^{s_{r}}}
$$

reduces to

$$
\begin{aligned}
& \zeta\left(s_{1}, s_{2}, s_{3}, \cdots, s_{r}\right)+\zeta\left(s_{2}, s_{1}, s_{3}, \cdots, s_{r}\right)+\zeta\left(s_{2}, s_{3}, s_{1}, \cdots, s_{r}\right)+\cdots \\
+ & \zeta\left(s_{1}+s_{2}, s_{3}, \cdots, s_{r}\right)+\zeta\left(s_{2}, s_{1}+s_{3}, \cdots, s_{r}\right)+\cdots \zeta\left(s_{2}, s_{3}, \cdots, s_{1}+s_{r}\right) .
\end{aligned}
$$

It should come as no surprise that this argument can be extended and in fact, the product of two MZV's of depth $r_{1}$ and $r_{2}$ is again positive integral linear combination of MZV's of depth at most $r_{1}+r_{2}$. These are called the "stuffle relations" and can be described as follows. In the notation introduced above, let $x_{j}$ denote the word $x^{j-1} y$. The "stuffle product" $*$ on $\mathbb{Q}\langle X\rangle$ is defined as follows:

$$
e * u=u * e=u
$$

for the empty word $e$ and all words $u \in X^{*}$;

$$
x_{j} u * x_{k} v=x_{j}\left(u * x_{k} v\right)+x_{k}\left(x_{j} u * v\right)+x_{j+k}(u * v)
$$

which differs from the shuffle relation in the last term. Then, it turns out that

$$
\zeta\left(w_{\alpha}\right) \zeta\left(w_{\beta}\right)=\zeta\left(w_{\alpha} * w_{\beta}\right) .
$$

In addition to these relations, and the shuffle relations

$$
\zeta\left(w_{\alpha}\right) \zeta\left(w_{\beta}\right)=\zeta\left(w_{\alpha} \amalg w_{\beta}\right),
$$

seen earlier, there is one more family of relations. They are all of the form

$$
\zeta\left(x \amalg w_{\alpha}\right)=\zeta\left(x * w_{\alpha}\right) .
$$

(We refer the reader to [40] for further details.) It is conjectured (see [43]) that these are the only relations amongs the MZV's. 
Zagier [41] has made the following more precise conjecture. Let $V_{k}$ be the $\mathbb{Q}$ vector subspace spanned by the MZV's of weight $k$. Set $V_{0}=\mathbb{Q}, V_{1}=\{0\}$. Using either the shuffle or stuffle relations, we see that

$$
V_{k} \cdot V_{k^{\prime}} \subseteq V_{k+k^{\prime}}
$$

If we denote by $V$ the $\mathbb{Q}$-vector space spanned by all the $V_{k}$ 's, then $V$ is a subalgebra of the reals over $\mathbb{Q}$ graded by the weight. Goncharov conjectures (see [39]) that

$$
V=\oplus_{k=0}^{\infty} V_{k}
$$

and Zagier predicts that if $d_{k}=\operatorname{dim} V_{k}$, then

$$
d_{k}=d_{k-2}+d_{k-3} \text {. }
$$

In other words, the Hilbert series of the graded algebra $V$ is completely determined:

$$
\sum_{k=0}^{\infty} d_{k} t^{k}=\frac{1}{1-t^{2}-t^{3}}
$$

This conjecture would imply the algebraic independence of $\pi, \zeta(3), \zeta(5), \ldots$ In a recent paper, Terasoma [37] as well as Deligne and Goncharov [16] proved using the theory of mixed Tate motives that

$$
\operatorname{dim} V_{k} \leq d_{k} .
$$

In $[\mathbf{1 6}]$, the authors consider the more general problem of the $\mathbb{Q}$-vector space spanned by all the values

$$
\sum_{n_{1}>n_{2}>\cdots>n_{r} \geq 1} \frac{\zeta_{1}^{n_{2}-n_{1}} \zeta_{2}^{n_{3}-n_{2}} \cdots \zeta_{r}^{-n_{r}}}{n_{1}^{s_{1}} n_{2}^{s_{2}} \cdots n_{r}^{s_{r}}}
$$

where $\zeta_{i}$ are fixed $N$-th roots of unity and $s_{1}, \ldots, s_{r}$ are positive integers with $s_{1} \neq 1$.

\section{A General Theorem}

We begin by proving the following theorem.

THEOREM 3.1. Let

$$
f(s)=\sum_{n=1}^{\infty} \frac{a_{n}}{n^{s}}
$$

be a Dirichlet series absolutely convergent in $\operatorname{Re}(s)>1$. Suppose $f(s)$ extends to a meromorphic function for all $s \in \mathbb{C}$. Then,

$$
f(s ; x):=\sum_{n=1}^{\infty} \frac{a_{n}}{(n+x)^{s}}
$$

extends to a meromorphic function for all $s \in \mathbb{C}$. Furthermore, the possible poles of $f(s ; x)$ are contained in the positive integral translates of the poles of $f(s)$. If $f(s)$ has a simple pole at $s=1$ with residue 1 , then $f(s ; x)$ also has only a simple pole at $s=1$ with residue 1 . If $f(s)$ extends to an entire function, then $f(s ; x)$ also extends to an entire function. 
Proof. Without loss of generality, we may suppose $0<|x|<1$. (If not, we may begin our summation of the Dirichlet series from $n_{0}$ with $n_{0}>|x|$.) For $\operatorname{Re}(s)>1$, we write our series as

$$
f(s ; x)=\sum_{n=1}^{\infty} \frac{a_{n}}{n^{s}}\left(1+\frac{x}{n}\right)^{-s} .
$$

We may expand the summand by using the binomial theorem and then interchange summations to get

$$
f(s ; x)=\sum_{r=0}^{\infty}\left(\begin{array}{c}
-s \\
r
\end{array}\right) f(s+r) x^{r} .
$$

The absolute convergence of this series is easily established using any of the standard tests. Indeed, for sufficiently large $r, f(s+r)$ is bounded. Applying the $r$-th root test together with the observation

$$
\log \left|\left(\begin{array}{c}
-s \\
r
\end{array}\right)\right| \leq \sum_{j=1}^{r} \log \left(1+\frac{|s|}{j}\right) \ll|s| \log r,
$$

shows that the series converges absolutely for $|x|<1$ and $\operatorname{Re}(s)>1$ since $f(s)$ is absolutely convergent there. In fact, we can say more. The summation from $r=1$ to infinity is absolutely convergent for $\operatorname{Re}(s)>0$. More generally, the summation from $r=M+1$ to infinity is absolutely convergent in the region $\operatorname{Re}(s)>-M$ for any integer $\geq 0$. As the sum from $r=0$ to $M$ is meromorphic, we deduce that $f(s ; x)$ is meromorphic for $\operatorname{Re}(s)>-M$. Since $M$ is arbitrary, this completes the proof of meromorphic continuation. We note in this argument, that if $f(s)$ extends to an entire function, so does $f(s ; x)$. The second part of the assertion of theorem is also clear since the possible poles of $f(s ; x)$ can only occur among the integer translates of the poles of $f(s)$.

We will refer to the method encoded in Theorem 3.1 as the binomial principle of analytic continuation. In the next two sections, we apply this theorem to study $\zeta(s), \zeta(s ; x)$ and more generally $\zeta\left(s_{1}, \ldots, s_{r} ; x_{1}, \ldots, x_{r}\right)$.

\section{The Hurwitz and Riemann zeta functions revisited}

Applying Theorem 3.1 with $f(s)$ equal to $\zeta(s)$, we find that

Proposition 4.1. For $0<x<1$,

$$
-\frac{1}{x^{s}}+\zeta(s, x)-\zeta(s)=\sum_{r=1}^{\infty}\left(\begin{array}{c}
-s \\
r
\end{array}\right) \zeta(s+r) x^{r} .
$$

REMARK 4.2. Observe that this identity gives immediately the analytic continuation of $\zeta(s, x)$ in an inductive way, once we know the continuation of $\zeta(s)$. Our point is that, in fact, the analytic continuation of $\zeta(s)$ can also be derived from it by considering $x=\frac{1}{2}$.

Notice that

$$
\begin{aligned}
\zeta\left(s, \frac{1}{2}\right) & =2^{s} \sum_{n=0}^{\infty} \frac{1}{(2 n+1)^{s}} \\
& =\left(2^{s}-1\right) \zeta(s) .
\end{aligned}
$$

Therefore, putting $x=\frac{1}{2}$ in the proposition, we obtain: 
THEOREM 4.3.

$$
\left(2^{s}-2\right) \zeta(s)=2^{s}+\sum_{r=1}^{\infty}\left(\begin{array}{c}
-s \\
r
\end{array}\right) 2^{-r} \zeta(s+r) .
$$

It is to be noted that recursions of this kind were also discovered by Ramaswami [33] in 1934 by a completely different method. The reader may also consult section 2.14 of $[\mathbf{3 8}]$, as well as [23], [22], and [14].

Thus, the theorem gives the analytic continuation of $\zeta(s)$ by induction. This is first valid in the half-plane of absolute convergence $\operatorname{Re}(s)>1$. The formula allows us to inductively obtain a meromorphic continuation of $\zeta(s)$. To be more precise, we first consider $\operatorname{Re}(s)>0$. Then, the right hand side is analytic there. Hence, $\left(2^{s}-2\right) \zeta(s)$ extends analytically to this region. This gives us a meromorphic continuation of $\zeta(s)$ for $\operatorname{Re}(s)>0$, with possible poles at

$$
s=1+\frac{2 \pi i m}{\log 2}, m \in \mathbb{Z} .
$$

To derive the complete analytic continuation, we observe the following. For any natural number $q$, we have

Proposition 4.4.

$$
\left(q^{s}-q\right) \zeta(s)=\sum_{a=1}^{q}\left[\zeta\left(s, \frac{a}{q}\right)-\zeta(s)\right] .
$$

Proof. We have

$$
q^{-s} \sum_{a=1}^{q} \zeta\left(s, \frac{a}{q}\right)=\sum_{a=1}^{q} \sum_{n=0}^{\infty} \frac{1}{(q n+a)^{s}}=\zeta(s),
$$

as the first summation on the right hand side is over a complete set of residue classes $(\bmod q)$ and the inner sum is

$$
\sum_{n \equiv a(\bmod q)}^{\infty} n^{-s}
$$

By Proposition 4.1, we have an analytic continuation of $\zeta\left(s, \frac{a}{q}\right)-\zeta(s)$ for $\operatorname{Re}(s)>0$, with possible poles at

$$
s=1+\frac{2 \pi i m}{\log q}, m \in \mathbb{Z}
$$

Taking $q=3$ and combining it with our remark before Proposition 4.4, we obtain that $\zeta(s)$ extends analytically for $\operatorname{Re}(s)>0$ except for a possible pole at an element of

$$
\left\{1+\frac{2 \pi i m}{\log 2}: m \in \mathbb{Z}\right\} \cap\left\{1+\frac{2 \pi i n}{\log 3}: n \in \mathbb{Z}\right\} .
$$

If $s_{0}$ is in the intersection, we must have $2^{n}=3^{m}$ for some $m, n \in \mathbb{Z}$. By unique factorization, the only solution is $m=n=0$. Thus, $\zeta(s)$ extends analytically for $\operatorname{Re}(s)>0$ apart from a possible pole at $s=1$. Now, by Theorem 4.3 , we have an 
analytic continuation of $\left(2^{s}-2\right) \zeta(s)$ for $\operatorname{Re}(s)>0$. Moreover, a simple calculation shows using Theorem 4.3

$$
\begin{aligned}
\lim _{s \rightarrow 1}\left(2^{s}-2\right) \zeta(s) & =2-\sum_{r=1}^{\infty}(-1)^{r} 2^{-r} \sum_{n=1}^{\infty} \frac{1}{n^{r+1}} \\
& =2-\sum_{n=1}^{\infty} \frac{1}{n} \sum_{r=1}^{\infty}\left(\frac{-1}{2 n}\right)^{r} \\
& =2-\sum_{n=1}^{\infty} \frac{1}{n(2 n+1)} \\
& =2-2 \sum_{n=1}^{\infty}\left(\frac{1}{2 n}-\frac{1}{2 n+1}\right) \\
& =2 \log 2 .
\end{aligned}
$$

But,

Therefore,

$$
\lim _{s \rightarrow 1} \frac{2^{s}-2}{s-1}=2 \log 2
$$

$$
\lim _{s \rightarrow 1}(s-1) \zeta(s)=\lim _{s \rightarrow 1} \frac{s-1}{2^{s}-2}\left(2^{s}-2\right) \zeta(s)=1
$$

and we deduce that $\zeta(s)$ has a simple pole at $s=1$, and is analytic for $\operatorname{Re}(s)>$ $0, s \neq 1$. Combining this with Theorem 4.3 and induction, we immediately deduce:

THEOREM 4.5. $\zeta(s)$ extends analytically for all $s \in \mathbb{C}$ except for a simple pole at $s=1$ where it has residue 1 .

Observe that from Proposition 4.3 and Theorem 4.5,

$$
\left(2^{s}-2\right) \zeta(s)=2^{s}-\frac{s \zeta(s+1)}{2}+\sum_{r=2}^{\infty}\left(\begin{array}{c}
-s \\
r
\end{array}\right) \zeta(s+r) 2^{-r}
$$

where we see the right hand side is analytic for $\operatorname{Re}(s)>-1$. We may substitute $s=0$ in the above formula and deduce that $\zeta(0)=-\frac{1}{2}$.

Using Proposition 4.1 and Theorem 4.5, we also obtain in a similar inductive fashion the analytic continuation of $\zeta(s, x)$.

TheOREM 4.6. $\zeta(s, x)$ extends analytically for all $s \in \mathbb{C}$ except for a simple pole at $s=1$ where it has residue 1 .

\section{Analytic continuation of multiple Hurwitz zeta functions}

Let us now consider the multiple Hurwitz zeta function. If we fix $s_{2}, \ldots, s_{r}$ and consider it as a function of $s_{1}$, then it is not difficult to see that the binomial principle allows us to extend the multiple Hurwitz zeta function to the entire complex plane as a meromorphic function of $s_{1}$. Thus,

$$
\zeta\left(s_{1}, s_{2}, \ldots, s_{r} ; x_{1}, \ldots, x_{r}\right)=\sum_{j=0}^{\infty}\left(\begin{array}{c}
-s_{1} \\
j
\end{array}\right) x_{1}^{j} \zeta\left(s_{1}+j, s_{2}, \ldots, s_{r} ; 0, x_{2}, \ldots, x_{r}\right) .
$$

Since the right hand side defines a meromorphic function of $s_{1}$ for $\operatorname{Re}\left(s_{1}\right)>0$, we can inductively derive the analytic continuation of $\zeta\left(s_{1}, \ldots, s_{r} ; x_{1}, \ldots, x_{r}\right)$ for $s_{2}, \ldots, s_{r}$ fixed. However, we would like to derive the continuation as a function in $\mathbb{C}^{r}$. 
For functions of several complex variables, a famous theorem of Hartogs [19] applies. This says that if we have a function of $r$ complex variables and we fix any $r-1$ of them and the resulting function is analytic in an open set $D \subseteq \mathbb{C}^{r}$, then the function itself is analytic as a function in $D$. We will apply this fact in dealing with the meromorphic continuation of the multiple Hurwitz zeta functions. We proceed by induction and take for granted that

$$
\zeta\left(s_{1}, \ldots, s_{r}\right)
$$

admits a meromorphic continuation to $\mathbb{C}^{r}$. We state this fact formally for future reference.

TheOrem 5.1. [42], [1] The multiple zeta function

$$
\zeta\left(s_{1}, \ldots, s_{r}\right)
$$

extends to a meromorphic function in $\mathbb{C}^{r}$ and has singularities on the hyperplanes

$$
s_{1}=1, \quad s_{1}+s_{2}=2,1,0,-2,-4, \ldots
$$

and for $j=3, \ldots, r$,

$$
s_{1}+s_{2}+\cdots s_{j} \in \mathbb{Z}_{\leq j}
$$

where $\mathbb{Z}_{\leq j}$ is the set of integers less than or equal to $j$.

A more precise statement of the previous theorem is the following which can be deduced from $[\mathbf{1}]$. In the region $\operatorname{Re}\left(s_{1}+\cdots+s_{r}\right)>-M$ (with $M$ a positive integer), there is a polynomial $P_{M}\left(s_{1}, \ldots, s_{r}\right)$ which is a product of distinct linear forms of the form

$$
s_{1}+s_{2}+\cdots+s_{j}-t
$$

with $1 \leq j \leq r$ and $t$ a positive integer $\leq j$ such that

$$
P_{M}\left(s_{1}, \ldots, s_{r}\right) \zeta\left(s_{1}, \ldots, s_{r}\right)
$$

is analytic there. Moreover, in this region, there are constants $A$ and $B$ (depending only on $M$ ) such that

$$
\left|P_{M}\left(s_{1}, \ldots, s_{r}\right) \zeta\left(s_{1}, \ldots, s_{r}\right)\right| \leq\left(\left|\operatorname{Im}\left(s_{1}\right)\right|+\cdots+\left|\operatorname{Im}\left(s_{r}\right)\right|+B\right)^{A} .
$$

This understanding will be implicit in our induction argument below where we derive the meromorphic continuation of the multiple Hurwitz zeta functions. The case $r=1$ is the classical theory of the Riemann and Hurwitz zeta functions discussed in the previous section. We therefore begin with the two variable case. Let us consider the series

$$
\zeta\left(s_{1}, s_{2} ; 0, x_{2}\right):=\sum_{n_{1}>n_{2} \geq 1} \frac{1}{n_{1}^{s_{1}}\left(n_{2}+x_{2}\right)^{s_{2}}}
$$

and applying the binomial expansion as before shows that this is equal to

$$
\sum_{j=0}^{\infty}\left(\begin{array}{c}
-s_{2} \\
j
\end{array}\right) x_{2}^{j} \zeta\left(s_{1}, s_{2}+j\right)
$$

and the summands on the right are the usual multiple zeta functions. Fixing $s_{1}$, and applying Theorem 5.1, we deduce that $\zeta\left(s_{1}, s_{2} ; 0, x_{2}\right)$ is a meromorphic function of $s_{2}$ by an application of Theorem 5.1. Fixing $s_{2}$, we see that it is a meromorphic 
function of $s_{1}$. Applying Hartogs' theorem, we get the meromorphic continuation of $\zeta\left(s_{1}, s_{2} ; 0, x_{2}\right)$. A similar reasoning applies to

$$
\zeta\left(s_{1}, s_{2} ; x_{1}, 0\right):=\sum_{n_{1}>n_{2} \geq 1} \frac{1}{\left(n_{1}+x_{1}\right)^{s_{1}} n_{2}^{s_{2}}} .
$$

If we fix $s_{2}$ and apply our binomial principle, we get the sum is

$$
\sum_{j=0}^{\infty}\left(\begin{array}{c}
-s_{1} \\
j
\end{array}\right) x_{1}^{j} \zeta\left(s_{1}+j, s_{2}\right)
$$

and the right hand side is meromorphic for all $s_{1}$. If we fix $s_{1}$, and consider it as a function of $s_{2}$, we obtain

$$
\zeta\left(s_{1}, s_{2} ; x_{1}, 0\right)=\sum_{n_{2} \geq 1} \frac{1}{n_{2}^{s_{2}}} \sum_{n_{1}>n_{2}} \frac{1}{\left(n_{1}+x_{1}\right)^{s_{1}}} .
$$

Upon writing the inner sum as

$$
\zeta\left(s_{1} ; x_{1}\right)-\sum_{n_{1} \leq n_{2}} \frac{1}{\left(n_{1}+x_{1}\right)^{s_{1}}}
$$

we deduce that

$$
\zeta\left(s_{1}, s_{2} ; x_{1}, 0\right)=\zeta\left(s_{1} ; x_{1}\right) \zeta\left(s_{2}\right)-\zeta\left(s_{2}, s_{1} ; 0, x_{1}\right)-\sum_{n_{2}=1}^{\infty} \frac{1}{n_{2}^{s_{2}}\left(n_{2}+x_{1}\right)^{s_{1}}} .
$$

The last series may be written

$$
\sum_{j=0}^{\infty}\left(\begin{array}{c}
-s_{1} \\
j
\end{array}\right) x_{1}^{j} \zeta\left(s_{1}+s_{2}+j\right)
$$

which again is analytic by our binomial principle.

Now we are ready to consider $\zeta\left(s_{1}, s_{2} ; x_{1}, x_{2}\right)$. Let us fix $s_{2}$. Then,

$$
\sum_{n_{1}>n_{2} \geq 1} \frac{1}{\left(n_{1}+x_{1}\right)^{s_{1}}\left(n_{2}+x_{2}\right)^{s_{2}}}=\sum_{j=0}^{\infty}\left(\begin{array}{c}
-s_{1} \\
j
\end{array}\right) x_{1}^{j} \zeta\left(s_{1}+j, s_{2} ; 0, x_{2}\right) .
$$

By our preceding discussion, the right hand side is a meromorphic function of $s_{1}$. If we fix $s_{1}$, then a similar analysis gives the meromorphic continuation of $\zeta\left(s_{1}, s_{2} ; x_{1}, x_{2}\right)$.

If we call the $x$-length of $\zeta\left(s_{1}, \ldots, s_{r} ; x_{1}, \ldots, x_{r}\right)$ to be the number of $x_{i}$ which are non-zero, then we may apply induction on the $x$-length to deduce the meromorphic continuation of $\zeta\left(s_{1}, \ldots, s_{r} ; x_{1}, \ldots, x_{r}\right)$. Indeed, fixing one of the variables and applying the binomial principle, we immediately deduce by induction the desired meromorphic continuation. For example,

$$
\zeta\left(s_{1}, \ldots, s_{r} ; x_{1}, \ldots, x_{r}\right)=\sum_{j=0}^{\infty}\left(\begin{array}{c}
-s_{r} \\
j
\end{array}\right) \zeta\left(s_{1}, \ldots, s_{r}+j ; x_{1}, \ldots, x_{r-1}, 0\right) .
$$

TheOREM 5.2. The multiple Hurwitz zeta function,

$$
\zeta\left(s_{1}, s_{2}, \ldots, s_{r} ; x_{1}, x_{2}, \ldots, x_{r}\right)
$$

extends to a meromorphic function in $\mathbb{C}^{r}$. 
The inductive principle applied in the proof of the above theorem also shows that for any positive integer $M$ and for $\operatorname{Re}\left(s_{1}+\cdots+s_{r}\right)>-M$, there is a polynomial $P_{M}\left(s_{1}, \ldots, s_{r}\right)$ which is a product of linear forms such that

$$
P_{M}\left(s_{1}, \ldots, s_{r}\right) \zeta\left(s_{1}, \ldots, s_{r} ; x_{1}, \ldots, x_{r}\right)
$$

is analytic in this region. Moreover, as before, a polynomial growth estimate of the form

$$
\left|P_{M}\left(s_{1}, \ldots, s_{r}\right) \zeta\left(s_{1}, \ldots, s_{r} ; x_{1}, \ldots, x_{r}\right)\right| \leq\left(\left|\operatorname{Im}\left(s_{1}\right)\right|+\cdots+\left|\operatorname{Im}\left(s_{r}\right)\right|+B\right)^{A}
$$

also holds in this region.

By a more careful analysis, it is possible to identify the pole set of the multiple Hurwitz zeta function. This has been done by Akiyama and Ishikawa and we state it for future reference.

Theorem 5.3. (Akiyama and Ishikawa) The multiple Hurwitz zeta function

$$
\zeta\left(s_{1}, \ldots, s_{r} ; x_{1}, \ldots, x_{r}\right)
$$

extends to a meromorphic function in $\mathbb{C}^{r}$ with possible singularities on

$$
s_{1}=1, \quad s_{1}+s_{2}+\cdots+s_{j} \in \mathbb{Z}_{\leq j}, \quad j=2,3, \ldots, r .
$$

If the $x_{i}$ are all rational, and $x_{2}-x_{1} \neq 0$ or $1 / 2$, then the above set coincides with the complete set of singularities. If $x_{2}-x_{1}=1 / 2$, then

$$
s_{1}=1, \quad s_{1}+s_{2}=2,0,-2,-4,-6, \ldots
$$

and for $3 \leq j \leq r$,

$$
s_{1}+s_{2}+\cdots+s_{j} \in \mathbb{Z}_{\leq j}
$$

forms the complete set of singularities. If $x_{2}-x_{1}=0$, then

$$
s_{1}=1, \quad s_{1}+s_{2}=2,1,0,-2,-4,-6, \cdots
$$

and for $3 \leq j \leq r$,

$$
s_{1}+s_{2}+\cdots+s_{r} \in \mathbb{Z}_{\leq j}
$$

forms the complete set of singularities.

We want to make some remarks concerning what we call quasi-multiple Hurwitz zeta functions. The summation condition on multiple zeta functions are of the form

$$
n_{1}>n_{2}>\cdots>n_{r} .
$$

Instead of considering the strict inequality condition, we may replace the condition with any of the possible $2^{r-1}$ variations, where equality is also allowed. A multiple Hurwitz zeta function with any of the possible $2^{r-1}$ such variations on the summation condition will be referred to as a quasi-multiple Hurwitz zeta function. Our point is that Theorem 5.2 allows us to deduce the meromorphic continuation of these cognate multiple Hurwitz zeta functions also. To see this, let us consider the simple case of the series corresponding to the condition

$$
n_{1} \geq n_{2}>\cdots>n_{r}
$$

It is clear that the series corresponding to this condition is the sum of the usual multiple Hurwitz zeta function and a series of the form

$$
\sum_{n_{1}>n_{3}>\cdots>n_{r}} \frac{1}{\left(n_{1}+x_{1}\right)^{s_{1}}\left(n_{1}+x_{2}\right)^{s_{2}} \cdots\left(n_{r}+x_{r}\right)^{s_{r}}} .
$$


This sum can be expanded as a double binomial series as

$$
\sum_{j=0}^{\infty} \sum_{k=0}^{\infty}\left(\begin{array}{c}
-s_{1} \\
j
\end{array}\right)\left(\begin{array}{c}
-s_{2} \\
k
\end{array}\right) x_{1}^{j} x_{2}^{k} \zeta\left(s_{1}+s_{2}+j+k, s_{3}, \ldots, s_{r} ; 0, x_{3}, \ldots, x_{r}\right)
$$

and the summands involve multiple zeta functions of depth $r-1$. One needs to apply growth estimates to establish convergence. Thus, we can derive the meromorphic continuation of the quasi-multiple Hurwitz zeta functions using a similar inductive principle as before. This remark will be used in the next section to derive the meromorphy of multiple $L$-functions.

As the referee has pointed out to us, the quasi-multiple Hurwitz zeta functions discussed above are a special case of a general multiple zeta function discussed by Matsumoto [26].

\section{Multiple $L$-functions}

As in $[\mathbf{2}]$, we can deduce the meromorphic continuation of multiple $L$-functions. However, it does not seem to be a simple matter to clearly describe the location of singularities. For characters $\chi_{1}, \ldots, \chi_{r}$ of modulus $q$, we begin by writing $L\left(s_{1}, \cdots, s_{r} ; \chi_{1}, \ldots, \chi_{r}\right)$ as

$$
q^{-s} \sum_{a_{1}, a_{2}, \ldots, a_{r}=1}^{q-1} \chi_{1}\left(a_{1}\right) \chi_{2}\left(a_{2}\right) \cdots \chi_{r}\left(a_{r}\right) \sum_{q n_{1}+a_{1}>q n_{2}+a_{2}>\cdots>q n_{r}+a_{r}} \frac{1}{\left(n_{1}+a_{1} / q\right)^{s_{1}}} \cdots \frac{1}{\left(n_{r}+a_{r} / q\right)^{s_{r}}},
$$

where $s=s_{1}+s_{2}+\cdots+s_{r}$. The condition of summation in the inner sum can be rewritten as

$$
n_{1}>n_{2}+\frac{a_{2}-a_{1}}{q}>\cdots>n_{r}+\frac{a_{r}-a_{r-1}}{q} .
$$

If each of the differences $a_{i}-a_{i-1}$ are non-negative, then, the summation condition is the same as

$$
n_{1}>n_{2}>\cdots>n_{r}
$$

so that the inner sum is the multiple Hurwitz zeta function

$$
\zeta\left(s_{1}, \ldots, s_{r} ; a_{1} / q, \cdots, a_{r} / q\right) \text {. }
$$

If any of the differences $a_{i}-a_{i-1}$ are negative, then the inner sum is a quasimultiple Hurwitz zeta function. By our remarks in the previous section, these admit a meromorphic continuation to $\mathbb{C}^{r}$ and thus, we obtain the desired meromorphic continuation of the multiple $L$-functions.

The precise location of the singularities of the multiple $L$-functions seems to be an open problem worthy of further research. The $r=1$ case is classical. The $r=2$ case was worked out in complete detail by Akiyama and Ishikawa [2]. We also point out that the problem of locating the singularities of $L\left(s, \ldots, s ; \chi_{1}, \ldots, \chi_{r}\right)$ is studied in [21].

\section{Special values of $\zeta(s, x)$}

In this section, we want to show that Theorems 4.3 and 4.1 can be used to derive the following classical results:

TheOREM 7.1. For any positive integer $k \geq 1$, we have

$$
\zeta(1-k)=(-1)^{k-1} \frac{B_{k}}{k} .
$$


More generally, we have:

THEOREM 7.2.

$$
\zeta(1-k, x)=-\frac{B_{k}(x)}{k}, k \geq 2 .
$$

We begin with the proof of Theorem 7.1. For $k=1$, we have already noted $\zeta(0)=-\frac{1}{2}$. We proceed by induction on $k$. We put $s=1-k$ in Theorem 4.3 and take into account that $\zeta(s+r)$ has a simple pole at $s=1-r$. Thus, we obtain the recurrence

$$
\left(2^{1-k}-2\right) \zeta(1-k)=2^{1-k}+\sum_{r=1}^{k-1}\left(\begin{array}{c}
k-1 \\
r
\end{array}\right) \frac{1}{2^{r}} \zeta(1-k+r)-\frac{1}{2^{k} k} .
$$

Then, by induction, we see that the right hand side becomes

$$
2^{1-k}+\sum_{r=1}^{k-1}\left(\begin{array}{c}
k-1 \\
r
\end{array}\right) \frac{1}{2^{r}}(-1)^{k-r-1} \frac{B_{k-r}}{k-r}-\frac{1}{2^{k} k} .
$$

This can be re-written as

$$
2^{1-k}+\frac{1}{k} \sum_{r=1}^{k-1}\left(\begin{array}{l}
k \\
r
\end{array}\right) \frac{1}{2^{r}}(-1)^{k-r-1} B_{k-r}-\frac{1}{2^{k} k}=2^{1-k}+\frac{1}{k} \sum_{r=0}^{k}\left(\begin{array}{l}
k \\
r
\end{array}\right) \frac{1}{2^{r}}(-1)^{k-r-1} B_{k-r}-\frac{1}{k}(-1)^{k-1} B_{k} .
$$

Thus, we get

$$
\left(2^{1-k}-2\right) \zeta(1-k)=2^{1-k}-(-1)^{k-1} \frac{B_{k}}{k}-\frac{1}{k} \sum_{r=0}^{k}\left(\begin{array}{l}
k \\
r
\end{array}\right) \frac{1}{2^{r}}(-1)^{k-r} B_{k-r} .
$$

To determine what the right hand side is, we consider

$$
\sum_{k=0}^{\infty} c_{k} \frac{x^{k}}{k !}:=\sum_{k=0}^{\infty} \frac{x^{k}}{2^{k} k !} \sum_{k=0}^{\infty}(-1)^{k} B_{k} \frac{x^{k}}{k !} .
$$

Then,

$$
c_{k}=\sum_{r=0}^{k}\left(\begin{array}{l}
k \\
r
\end{array}\right) \frac{1}{2^{r}}(-1)^{k-r} B_{k-r}
$$

for every $k \geq 1$. Note that

$$
\sum_{k=0}^{\infty} \frac{c_{k}}{k !} x^{k}=\frac{e^{x / 2} x}{1-e^{-x}}
$$

This can be rewritten as

$$
x e^{x / 2}+\frac{x}{e^{x / 2}-e^{-x / 2}} .
$$

The coefficient of $x^{k} / k$ ! in the first term is $k / 2^{k}$. To determine the corresponding coefficient in the second term, we write it as

$$
\frac{i x}{2 \sin (i x / 2)} \text {. }
$$

The power series expression for the last term is well-known. Recall from [15] (page 88)that

$$
\frac{1}{\sin x}=x^{-1}+\sum_{m \geq 1} B_{2 m}(-1)^{m+1}\left(2^{2 m}-2\right) \frac{x^{2 m-1}}{(2 m) !}
$$


Using this formula, we can deduce that

$$
\frac{i x / 2}{\sin (i x / 2)}=1-\sum_{k=1}^{\infty} B_{2 k}\left(2^{2 k}-2\right)\left(\frac{x}{2}\right)^{2 k} \frac{1}{(2 k) !}
$$

Thus, if $k$ is even,

$$
c_{k}=\frac{k}{2^{k-1}}-B_{k} \frac{2^{k}-2}{2^{k}} .
$$

As a result,

$$
\left(2^{1-k}-2\right) \zeta(1-k)=2^{1-k}-(-1)^{k-1} \frac{B_{k}}{k}-\frac{1}{k}\left\{\frac{k}{2^{k-1}}-B_{k} \frac{\left(2^{k}-2\right)}{2^{k}}\right\}=\left(2-2^{1-k}\right) \frac{B_{k}}{k} .
$$

That is, $\zeta(1-k)=-B_{k} / k$. If $k$ is odd,

$$
c_{k}=\frac{k}{2^{k-1}}
$$

Thus

$$
\left(2^{1-k}-2\right) \zeta(1-k)=(-1)^{k} \frac{B_{k}}{k} .
$$

In particular, if $k=1$, then $\zeta(0)=-\frac{1}{2}=B_{1}$. If $k \geq 3$, and $k$ is odd, then $\zeta(1-k)=0=B_{k}$. The result now follows from the different cases considered above. The completes the proof of Theorem 7.1.

To prove Theorem 7.2, we have by Proposition 4.1

$$
\frac{-1}{x^{s}}+\zeta(s, x)-\zeta(s)=\sum_{r=1}^{\infty}(-1)^{r} \frac{s(s+1) \ldots(s+r-1)}{r !} \zeta(s+r) x^{r} .
$$

In the above equation, put $s=1-k$ and observe that if $r>k$, then the sum vanishes, since $\zeta(1-k+r)$ is analytic and the binomial coefficient vanishes.

At $r=k, \zeta(1-k+r)$ has a simple pole. Thus,

$$
\lim _{r \rightarrow k}(r-k) \zeta(1-k+r)=1
$$

This implies that

$$
\lim _{r \rightarrow k}(-1)^{r} \frac{(1-k)(2-k) \ldots(r-1-k)(r-k)}{r !} \zeta(1-k+r)=\frac{(-1)^{k}}{k !}(-1)^{k-1}(k-1) !=-\frac{1}{k} .
$$

So,

$$
-\frac{1}{x^{1-k}}+\zeta(1-k, x)-\zeta(1-k)=\sum_{r=1}^{k-1}\left(\begin{array}{c}
k-1 \\
r
\end{array}\right) \zeta(1-k+r) x^{r}-\frac{x^{k}}{k}
$$


Thus,

$$
\begin{aligned}
\zeta(1-k, x) & =x^{k-1}+\zeta(1-k)+\sum_{r=1}^{k-1}\left(\begin{array}{c}
k-1 \\
r
\end{array}\right) \zeta(1-k+r) x^{r}-\frac{x^{k}}{k} \\
& =x^{k-1}+\zeta(1-k)-\sum_{r=1}^{k-2}\left(\begin{array}{c}
k-1 \\
r
\end{array}\right) \frac{B_{k-r}}{k-r} x^{r}+\zeta(0) x^{k-1}-\frac{x^{k}}{k} \\
& =\frac{x^{k-1}}{2}+\zeta(1-k)-\frac{1}{k} \sum_{r=1}^{k-2}\left(\begin{array}{c}
k \\
r
\end{array}\right) B_{k-r} x^{r}-\frac{x^{k}}{k} \\
& =-B_{1} x^{k-1}-\frac{B_{k}}{k}-B_{0} \frac{x^{k}}{k}-\frac{1}{k} \sum_{r=1}^{k-2}\left(\begin{array}{c}
k \\
r
\end{array}\right) B_{k-r} x^{r} \\
& =-\frac{1}{k} \sum_{r=0}^{k}\left(\begin{array}{c}
k \\
r
\end{array}\right) B_{k-r} x^{r} \\
& =-\frac{B_{k}(x)}{k}
\end{aligned}
$$

The idea of this proof originates in $[\mathbf{3 1}]$. For a related derivation, see [36].

\section{Poly-Hurwitz Zeta Functions}

The series, which we met in the analysis of the previous sections,

$$
Z\left(s_{1}, s_{2} ; x_{1}, x_{2}\right):=\sum_{n=1}^{\infty} \frac{1}{\left(n+x_{1}\right)^{s_{1}}\left(n+x_{2}\right)^{s_{2}}}
$$

already arises in several works (see for example, [24]). Our method of analytic continuation of $\zeta(s)$ will also apply to poly-Hurwitz zeta functions which we define as follows:

$$
Z\left(s_{1}, s_{2}, \ldots, s_{r} ; x_{1}, x_{2}, \ldots, x_{r}\right)=\sum_{n=1}^{\infty}\left(n+x_{1}\right)^{-s_{1}} \ldots\left(n+x_{r}\right)^{-s_{r}}
$$

for $0<x_{i} \leq 1$. This series absolutely converges when $\operatorname{Re}\left(s_{1}+s_{2}+\cdots+s_{r}\right)>1$. Then, using our binomial principle,

$$
Z\left(s_{1}, s_{2}, \ldots, s_{r} ; x_{1}, x_{2}, \ldots, x_{r}\right)=\sum_{j_{1}, \ldots, j_{r}}\left(\begin{array}{c}
-s_{1} \\
j_{1}
\end{array}\right)\left(\begin{array}{c}
-s_{2} \\
j_{2}
\end{array}\right) \ldots\left(\begin{array}{c}
-s_{r} \\
j_{r}
\end{array}\right) x_{1}^{j_{1}} \ldots x_{r}^{j_{r}} \zeta\left(s_{1}+j_{1}+\cdots+s_{r}+j_{r}\right) .
$$

Using methods similar to those used in previous sections, we can obtain a meromorphic continuation of this function to $\mathbb{C}^{r}$.

The values

$$
Z\left(1-k_{1}, 1-k_{2}, \ldots, 1-k_{r} ; x_{1}, x_{2}, \ldots, x_{r}\right)
$$

can also be expressed as a polynomial in $x_{1}, \ldots, x_{r}$ with rational coefficients involving Bernoulli numbers. For example, let $r=2$. We will try to find the value of

$$
Z\left(s_{1}, s_{2} ; x_{1}, x_{2}\right)
$$


when $s_{i}=1-k_{i}, k_{i} \in \mathbb{Z}, k_{i} \geq 1$. By the above calculation,

$$
\begin{aligned}
Z\left(1-k_{1}, 1-k_{2} ; x_{1}, x_{2}\right) & =\sum_{j_{1}=0}^{k_{1}-1} \sum_{j_{2}=0}^{k_{2}-1}\left(\begin{array}{c}
k_{1}-1 \\
j_{1}
\end{array}\right)\left(\begin{array}{c}
k_{2}-1 \\
j_{2}
\end{array}\right) x_{1}^{j_{1}} x_{2}^{j_{2}} \zeta\left(1-k_{1}+1-k_{2}+j_{1}+j_{2}\right) \\
& =-\sum_{j_{1}=0}^{k_{1}-1} \sum_{j_{2}=0}^{k_{2}-1}\left(\begin{array}{c}
k_{1}-1 \\
j_{1}
\end{array}\right)\left(\begin{array}{c}
k_{2}-1 \\
j_{2}
\end{array}\right) x_{1}^{j_{1}} x_{2}^{j_{2}} \frac{B_{l\left(j_{1}, j_{2}\right)}}{l\left(j_{1}, j_{2}\right)},
\end{aligned}
$$

where $l\left(j_{1}, j_{2}\right)=k_{1}+k_{2}-1-j_{1}-j_{2}$. More generally, the above argument shows

$$
Z\left(1-k_{1}, 1-k_{2}, \ldots, 1-k_{r} ; x_{1}, x_{2}, \ldots, x_{r}\right)
$$

is a polynomial in $x_{1}, x_{2}, \ldots, x_{r}$ with rational coefficients given by Bernoulli numbers.

\section{Multiple Hurwitz zeta functions at complex arguments}

We now make a few final remarks about how $\zeta(s, x)$ and more generally, $\zeta\left(s_{1}, \ldots, s_{r} ; x_{1}, \ldots, x_{r}\right)$ can be studied for complex values of $x_{i}$. We begin with

$$
\zeta(s, z)=\sum_{n=0}^{\infty} \frac{1}{(n+z)^{s}}
$$

where $z$ is a complex number. The summand is to be interpreted as

$$
\exp (-s \log (n+z))
$$

and therefore, for the logarithm to be defined, we need to have $z$ not lie on the negative real axis. Analogous to the proof of Theorem 4.1, we note that

$$
\frac{-1}{z^{s}}+\zeta(s, z)-\zeta(s)=\sum_{n=1}^{\infty}\left(\frac{1}{(n+z)^{s}}-\frac{1}{n^{s}}\right) .
$$

Writing the summand as

$$
\frac{1}{n^{s}}\left(\left(1+\frac{z}{n}\right)^{-s}-1\right)
$$

and observing that the binomial theorem holds for all complex numbers with absolute value less than 1 , we obtain the following theorem.

THEOREM 9.1. Consider the cut unit disc

$$
\mathbb{D}=\{z:|z|<1\} \backslash\{z \in \mathbb{R}:-1<z \leq 0\} .
$$

The function $\zeta(s, z)$ for $z \in \mathbb{D}$ is analytic in the whole complex plane except for a simple pole at $s=1$.

Hence, by induction, we can derive the analytic continuation of $\zeta(s, z)$ for $z \in \mathbb{D}$ to the whole complex plane apart from a simple pole at $s=1$. This analysis can be extended to a wider region of $z$-values. For given any non-zero complex number $z$ not lying in the negative real axis, we can find a positive intger $m$ such that $|z|<m$. Now,

$$
-\frac{1}{z^{s}}+\zeta(s, z)-\zeta(s)=\sum_{n=1}^{m-1} \frac{1}{n^{s}}\left(\left(1+\frac{z}{n}\right)^{-s}-1\right)+\sum_{n=m}^{\infty} \frac{1}{n^{s}}\left(\left(1+\frac{z}{n}\right)^{-s}-1\right)
$$

For $|z|<m$, we have $|z / n|<1$, for all $n \geq m$. Thus, applying binomial theorem to the second term on the right hand side and by a change in the order of summation, we obtain a modification of Theorem 9.1. 
THEOREM 9.2. Let

$$
\mathbb{D}^{\prime}=\{z \in \mathbb{C}\} \backslash\{z \in \mathbb{R}: z \leq 0\} .
$$

Then, given $z \in \mathbb{D}^{\prime}, \exists m \in \mathbb{N}$ such that

$$
-\frac{1}{z^{s}}+\zeta(s, z)-\zeta(s)=\sum_{n=1}^{m-1} \frac{1}{n^{s}}\left(\left(1+\frac{z}{n}\right)^{-s}-1\right)+\sum_{r=1}^{\infty}\left(\begin{array}{c}
-s \\
r
\end{array}\right) z^{r}\left(\zeta(s+r)-\sum_{n=1}^{m-1} \frac{1}{n^{s+r}}\right) .
$$

Since the right hand side is analytic for $\operatorname{Re}(s)>0$, we obtain an analytic continuation of $\zeta(s, z)$ in this region. Thus, by induction, we can derive that the function $\zeta(s, z)$, for $z \in \mathbb{D}^{\prime}$ is analytic in the whole complex plane except for a simple pole at $s=1$.

It is now natural to inquire with this extended definition of the Hurwitz zeta function for complex values whether the analogue of Theorem 7.2 holds. This is indeed the case. We now prove the following result:

TheOREM 9.3. For $z \in \mathbb{D}^{\prime}$,

$$
\zeta(1-k, z)=-\frac{B_{k}(z)}{k}, k \in \mathbb{Z}, k \geq 2 .
$$

Proof. The result is clear for $z \in \mathbb{D}$ since the argument is identical to the proof of Theorem 7.2. More generally, let $z \in \mathbb{D}^{\prime}$. We have

$$
\begin{aligned}
-\frac{1}{z^{s}}+\zeta(s, z)-\zeta(s) & =\sum_{n=1}^{m-1} \frac{1}{n^{s}}\left(\left(1+\frac{z}{n}\right)^{-s}-1\right)+\sum_{r=1}^{\infty}\left(\begin{array}{c}
-s \\
r
\end{array}\right) z^{r} \zeta(s+r) \\
& -\sum_{r=1}^{\infty}\left(\begin{array}{c}
-s \\
r
\end{array}\right) z^{r}\left(\sum_{n=1}^{m-1} \frac{1}{n^{s+r}}\right) .
\end{aligned}
$$

In the above equation, we put $s=1-k$ where $k \in \mathbb{Z}, k \geq 2$. Then, we get the following equation :

$$
\begin{aligned}
-z^{k-1}+\zeta(1-k, z)-\zeta(1-k) & =\sum_{n=1}^{m-1} \frac{1}{n^{1-k}}\left(\left(1+\frac{z}{n}\right)^{k-1}-1\right)+\sum_{r=1}^{k-1}\left(\begin{array}{c}
k-1 \\
r
\end{array}\right) \zeta(1-k+r) z^{r} \\
& -\frac{z^{k}}{k}-\sum_{r=1}^{k-1}\left(\begin{array}{c}
k-1 \\
r
\end{array}\right) z^{r} \sum_{n=1}^{m-1} \frac{1}{n^{1-k+r}} .
\end{aligned}
$$

Now, the first term on the right hand side is

$$
\begin{aligned}
\sum_{n=1}^{m-1} \frac{1}{n^{1-k}}\left[\sum_{r=0}^{k-1}\left(\begin{array}{c}
k-1 \\
r
\end{array}\right)\left(\frac{z}{n}\right)^{r}-1\right] & =\sum_{n=1}^{m-1} \frac{1}{n^{1-k}}\left[\sum_{r=1}^{k-1}\left(\begin{array}{c}
k-1 \\
r
\end{array}\right)\left(\frac{z}{n}\right)^{r}\right] \\
& =\sum_{r=1}^{k-1}\left(\begin{array}{c}
k-1 \\
r
\end{array}\right) z^{r} \sum_{n=1}^{m-1} \frac{1}{n^{1-k+r}}
\end{aligned}
$$

Thus, this is cancelled by the last term and the proof is complete.

As the referee remarks, the last theorem is also clear by analytic continuation in the variable $z$. The same technique can be applied to study $Z\left(s_{1}, \ldots, s_{r} ; z_{1}, \ldots, z_{r}\right)$ and $\zeta\left(s_{1}, \ldots, s_{r} ; z_{1}, \ldots, z_{r}\right)$ with $z_{i} \in \mathbb{D}^{\prime}$. This topic has also been studied by Matsumoto $[\mathbf{2 7}]$. 


\section{Concluding Remarks}

The arithmetic nature of multiple $L$-values as well as conjectures concerning their algebraic independence seems to not have been investigated in the literature. Clearly, things become more subtle in this realm. For one thing, many of these multiple $L$-values make sense with $s_{1}=1$. In the $r=1$ case, these values involve regulator terms such as logarithms of fundamental units. It seems difficult at this point to make precise conjectures in the spirit of Zagier or Goncharov.

The question of precise determination of the singularities of multiple $L$-functions is still unresolved for $r \geq 3$. This looks like a very delicate problem requiring further analysis.

There is also one more point worth noting. The shuffle identities seem to apply only to multiple zeta values whereas the stuffle identities hold for multiple zeta functions also.

Acknowledgments. We would like to thank Professors David Bradley and Pierre Deligne for their comments on an earlier version of this paper. We are also grateful to the referee for providing us with additional references relevant to the topics discussed in this paper.

\section{References}

[1] S. Akiyama, S. Egami and Y. Tanigawa, Analytic continuation of multiple zeta functions and their values at non-positive integers, Acta Arithmetica, 98 (2001), no. 2, 107-116.

[2] S. Akiyama and H. Ishikawa, On analytic continuation of multiple $L$-functions and related zeta functions, Analytic Number Theory (Beijing/Kyoto, 1999), 1-16, Dev. Math., 6 Kluwer Acad. Publ., Dodrecht, 2002.

[3] T. Arakawa and M. Kaneko, Multiple zeta values, poly-Bernoulli numbers and related zeta functions, Nagoya Math. J., 153 (1999), 189-209.

[4] R. Apéry, Irrationalité de $\zeta(2)$ et $\zeta(3)$, Astérisque, 61 (1979), 11-13.

[5] T. Apostol and T.H. Vu, Dirichlet series related to the Riemann zeta function, Journal of Number Theory, 19 (1984), 85-102.

[6] F.V. Atkinson, The mean value of the Riemann zeta function, Acta Math., 81 (1949), 353-376.

[7] K. Ball and T. Rivoal, Irrationalité d'une infinité de valeurs de la fonction zêta aux entiers impairs, Inventiones Math., 146 (2001), no. 1, 193-207.

[8] J.M. Borwein, D.M. Bradley and D.J. Broadhurst, Evaluation of $k$-fold Euler/Zagier sums: a compendium of results for arbitrary $k$, Electronic Journal of Combinatorics, 4, No. 2, (1997), \# R5.

[9] J.M. Borwein, D.M. Bradley, D.J. Broadhurst, and P. Lisonek, Combinatorial aspects of multiple zeta values, Electronic Journal of Combinatorics, 5 (1998), no. 1, \# R 38.

[10] R. de la Bretèche, Estimation de sommes multiples de fonctions arithmétiques, Compositio Math., 128 (2001), no. 3, 261-298.

[11] P. Cartier, Fonctions polylogarithms, nombres polyzetas et groupes pro-unipotents, Séminaire Bourbaki, Vol. 2000/2001, Astérisque, 282 (2002), 137-173.

[12] P. Cartier, A mad day's work: from Grothendieck to Connes and Kontsevich. The evolution of concepts of space and symmetry, Bulletin of the Amer. Math. Soc., 38 (2001), no. 4, 389-408.

[13] K.T. Chen, Iterated path integrals, Bulletin of the Amer. Math. Soc., 83 (1977), 831-879.

[14] J. Choi and H.M. Srivastava, Series associated with the zeta and related functions, Kluwer Academic Publishers, Dordrecht, Boston, London, 2001.

[15] L. Comtet, Advanced Combinatorics, D. Reidel Publishing Company, 1974.

[16] P. Deligne and A. Goncharov, Groupes fondamentaux motiviques de Tate mixte, Ann. Scient. Éc. Norm. Sup., 38. 4th Series, (2005), 1-56. 
[17] A. Goncharov, Multiple polylogarithms, cyclotomy, and modular complexes, Math. Res. Letters, 5 (4) (1998), 569-618.

[18] M. Hoffman, Multiple harmonic series, Pacific Journal of Math., 152 (1992), no. 2, 275290 .

[19] L. Hörmander, An introduction to complex analysis in several variables, Third Edition, North-Holland Mathematical Library, 7, North-Holland Publishing Company, Amsterdam, 1990.

[20] A. Hurwitz, Mathematische Werke, Vol. 2, Basel, Birkhäuser, 1932.

[21] H. Ishikawa, On analytic properties of a multiple $L$-function, in Analytic extension formulas and their applications, (Fukuoka, 1999/Kyoto, 2000), 105-122, Int. Soc. Analytic Appl. Comput., 9, KluwerAcad. Publ., Dordrecht, 2001.

[22] S. Kanemitsu, M. Katsurada, and M. Yoshimoto, On the Hurwitz-Lerch zeta function, Aequationes Math., 59 (2000), no. 1-2, 1-19.

[23] M. Katsurada, Power series with the Riemann zeta function in the coefficients, Proc. Japan Acad. Ser. A. Math. Sci. 72 (1996), no. 3, 61-63.

[24] R. Masri, The Herglotz-Zagier function, double zeta functions and values of $L$-series, Journal of Number Theory, 106 (2004), 219-237.

[25] K. Matsumoto, On the analytic continuation of various multiple zeta functions, in Number Theory for the Millenium II, edited by M. A. Bennett et al, pp. 417-440, 2002, A. K. Peters.

[26] K. Matsumoto, On Mordell-Tornheim and other multiple zeta functions, Proceedings of the Session in Analytic Number Theory and Diophantine Equations, Bonner Math. Schriften, 360 (2003), 1-17.

[27] K. Matsumoto, The analytic continuation and the asymptotic behaviour of certain multiple zeta functions, I, Journal of Number Theory, 101 (2003), no. 2, 223-243.

[28] K. Matsumoto and Y. Tanigawa, The analytic continuation and the order estimate of multiple Dirichlet series, J. Théor. Nombres Bordeaux, 15 (2003), no. 1, 267-274.

[29] Y. Matsuoka, On the values of a certain Dirichlet series at rational integers, Tokyo Journal of Mathematics, 5 (1982), 399-403.

[30] N. Minh, G. Jacob, M. Petitot, N. Oussous, De l'algèbre des $\zeta$ de Riemann multivariées à l'algèbre des $\zeta$ de Hurwitz multivariées, Sem. Lothar. Combin. 44 (2000), Art. B44i, 21p.

[31] M. Ram Murty and M. Reece, A simple derivation of $\zeta(1-k)=-B_{k} / k$, Functiones et Approximatio, 28(2000), 141-154.

[32] M. Ram Murty, Introduction to $p$-adic analytic number theory, Studies in Advanced Mathematics, 27, 2002.

[33] V. Ramaswami, A note on Riemann's $\zeta$-function, Journal of the London Math. Soc., 9 (1934), 165-169.

[34] B. Riemann, Über die Anzahl der primzahlen unter einer gegebenen Grösse, Monatsber. Akadem. Berlin, (1859), 671-680.

[35] T. Rivoal, La fonction zêta de Riemann prend une infinité de valeurs irrationnelles aux entiers impairs, C.R. Acad. Sci. Paris Sér. I Math., 331:4 (2000), 267-270.

[36] H. M. Stark, Dirichlet's class number formula revisited, Contemporary Mathematics, 143 (1993), 571-577.

[37] T. Terasoma, Mixed Tate motives and multiple zeta values, Inventiones Math., 149 (2002), no. 2, 339-369.

[38] E.C. Titchmarsh, The Theory of the Riemann Zeta Function, (Revised by D.R. HeathBrown), Second Edition, Clarendon Press, 1986, Oxford.

[39] M. Waldschmidt, Valeurs zeta multiples. Une introduction, Journal de Théories des Nombres de Bordeaux, 12 (2000), 581-595.

[40] M. Waldschmidt, Multiple polylogarithms: an introduction, in Number Theory and Discrete Mathematics, Hindustan Book Agency and Birkhäuser-Verlag, 2002, 1-12.

[41] D. Zagier, Values of zeta functions and their applications, First European Congress of Mathematics, Vol. 2, Paris, 1992, pp. 497-512.

[42] J. Zhao, Analytic continuation of multiple zeta functions, Proceedings of the Amer. Math. Society, 128 (1999), No. 5, 1275-1283.

[43] V. V. Zudilin, Algebraic relations for multiple zeta values, Russian Math. Surveys, 58 (2003).

[44] V.V. Zudilin, One of the eight numbers $\zeta(5), \zeta(7), \ldots, \zeta(17), \zeta(19)$ is irrational, Mat. $Z a-$ metki, 70 (2001), 147-148, translation in Math. Notes, 70 (2001), no. 3-4, 426-431. 
Department of Mathematics, Queen's University, Kingston, Ontario, K7L 3N6, Canada

E-mail address: murty@mast.queensu.ca

Department of Mathematics, Queen's University, Kingston, Ontario, K7L 3N6, Canada

E-mail address: skaneen@mast.queensu.ca 